\title{
A DFT Investigation on the Mechanism of the Deacetylation Reaction Catalyzed by LpxC
}

\author{
Jesse J. Robinet and James W. Gauld \\ Department of Chemistry and Biochemistry, University of Windsor, Windsor, Ontario, N9B 3P4, \\ Canada
}

Supporting Information

(Table $\mathrm{S} 1$ and $\mathrm{S} 2 ; 36$ pages in total) 
Table S1. Full citation for Gaussian 03 (reference 28).

Frisch, M. J.; Trucks, G. W.; Schlegel, H. B.; Scuseria, G. E.; Robb, M. A.; Cheeseman, J. R.; Montgomery, Jr., J. A.; Vreven, T.; Kudin, K. N.; Burant, J. C.; Millam, J. M.; Iyengar, S. S.; Tomasi, J.; Barone, V.; Mennucci, B.; Cossi, M.; Scalmani, G.; Rega, N.; Petersson, G. A.; Nakatsuji, H.; Hada, M.; Ehara, M.; Toyota, K.; Fukuda, R.; Hasegawa, J.; Ishida, M.; Nakajima, T.; Honda, Y.; Kitao, O.; Nakai, H.; Klene, M.; Li, X.; Knox, J. E.; Hratchian, H. P.; Cross, J. B.; Bakken, V.; Adamo, C.; Jaramillo, J.; Gomperts, R.; Stratmann, R. E.; Yazyev, O.; Austin, A. J.; Cammi, R.; Pomelli, C.; Ochterski, J. W.; Ayala, P. Y.; Morokuma, K.; Voth, G. A.; Salvador, P.; Dannenberg, J. J.; Zakrzewski, V. G.; Dapprich, S.; Daniels, A. D.; Strain, M. C.; Farkas, O.; Malick, D. K.; Rabuck, A. D.; Raghavachari, K.; Foresman, J. B.; Ortiz, J. V.; Cui, Q.; Baboul, A. G.; Clifford, S.; Cioslowski, J.; Stefanov, B. B.; Liu, G.; Liashenko, A.; Piskorz, P.; Komaromi, I.; Martin, R. L.; Fox, D. J.; Keith, T.; Al-Laham, M. A.; Peng, C. Y.; Nanayakkara, A.; Challacombe, M.; Gill, P. M. W.; Johnson, B.; Chen, W.; Wong, M. W.; Gonzalez, C.; Pople, J. A. Gaussian 03, Gaussian, Inc., Wallingford CT, 2004.

Table S2. Optimized xyz-coordinates obtained at the PB-PCM-B3LYP/LACVP** level of theory and solution phase energies (h) obtained at the PB-PCM-B3LYP/LACV3P+**//PB-PCMB3LYP/LACVP** level of theory for all species considered in this present study.

\section{$\mathrm{H}_{2} \mathrm{O}$}

Solution phase energy: $\quad-76.46900131933$

$\begin{array}{llll}\mathrm{O} & 0.0000000000 & 0.0000000000 & -0.0669655491\end{array}$

$\mathrm{H} \quad 0.0000000000 \quad 0.7591494835 \quad 0.5313959303$

$\mathrm{H} \quad 0.0000000000 \quad-0.7591494835 \quad 0.5313959303$ 


\section{N-Methylacetamide}

\begin{tabular}{|c|c|c|c|}
\hline \multicolumn{2}{|c|}{ Solution phase energy: } & \multicolumn{2}{|c|}{-248.61770032722} \\
\hline $\mathrm{C}$ & 0.4814230432 & 0.1590689910 & 0.0003978637 \\
\hline 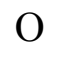 & 0.4217876667 & 1.3915164827 & 0.0009016743 \\
\hline & 1.8168071722 & -0.5660886679 & -0.0004179770 \\
\hline & -0.6230614303 & -0.6332796772 & -0.0000033720 \\
\hline & 2.3867273390 & -0.2538256207 & 0.8806342596 \\
\hline & 1.7250332392 & -1.6555275057 & -0.0001795039 \\
\hline & 2.3852162888 & -0.2541338022 & -0.8825309027 \\
\hline & -0.5001430905 & -1.6359232291 & -0.0009411413 \\
\hline & -1.9749509488 & -0.1003760945 & -0.0006388779 \\
\hline & -1.9028383392 & 0.9875765055 & -0.0011181231 \\
\hline & -2.5282778625 & -0.4206254682 & 0.8893207593 \\
\hline & -2.5275179179 & -0.4212852566 & -0.8908671981 \\
\hline
\end{tabular}

$\mathbf{1}_{\mathrm{aq}}$

Solution phase energy: $\quad-401.56681608395$

$\begin{array}{llll}\text { C } & 0.7377863010 & -0.3321419962 & 0.2923944120\end{array}$

$\begin{array}{llll}\text { O } & 0.3063453323 & 0.7864438888 & 0.6311191681\end{array}$

$\begin{array}{llll}\text { C } & -0.0950080761 & -1.5845288525 & 0.4554590364\end{array}$

$\begin{array}{llll}\mathrm{N} & 1.9559009904 & -0.4757893491 & -0.2661643691\end{array}$

$\begin{array}{llll}\mathrm{H} & 0.4453805319 & -2.4997328605 & 0.2006994482\end{array}$

$\begin{array}{llll}\mathrm{H} & -0.4437381352 & -1.6510823619 & 1.4905017458\end{array}$

H $\quad-0.9718814879 \quad-1.4809543430 \quad-0.1941203440$

$\begin{array}{llll}\mathrm{H} & 2.2797038884 & -1.4064097943 & -0.4881629337\end{array}$

$\begin{array}{llll}\text { C } & 2.8344672486 & 0.6529794263 & -0.5433377938\end{array}$

$\begin{array}{llll}\mathrm{H} & 3.4913946544 & 0.3993534483 & -1.3781915040\end{array}$ 


$\begin{array}{llll}\mathrm{H} & 3.4491905780 & 0.9175796211 & 0.3253171129 \\ \mathrm{H} & 2.2302864555 & 1.5215479799 & -0.8119922186 \\ \mathrm{O} & -2.5180172146 & -0.1134805510 & -1.4913588974 \\ \mathrm{O} & -2.3818601330 & 0.9214631169 & 1.0784000488 \\ \mathrm{H} & -2.6223451651 & 0.2472074942 & 1.7283307526 \\ \mathrm{H} & -1.3958800304 & 0.9049918620 & 1.0381759308 \\ \mathrm{H} & -2.5987052282 & 0.2694131760 & -0.5900216729 \\ \mathrm{H} & -1.9983477540 & 0.5451100058 & -1.9707947030\end{array}$

\section{TSC $_{\text {aq }}$}

Solution phase energy: $\quad-401.50001495178$

$\begin{array}{llll}\mathrm{C} & 0.9146934984 & -0.2593583853 & -0.0743743168 \\ \mathrm{O} & 1.8960429159 & 0.1522958383 & 0.5119318567 \\ \mathrm{O} & -0.5230719973 & -0.6826983781 & 1.3284547231 \\ \mathrm{H} & -0.3304013355 & -1.5859393544 & 1.6171483361 \\ \mathrm{H} & -1.0713968891 & 0.3706416127 & -0.6710437796 \\ \mathrm{~N} & -0.0346044598 & 0.7867036685 & -0.6974211457 \\ \mathrm{H} & 0.2538212352 & 0.9171389541 & -1.6713294262 \\ \mathrm{C} & 0.0002480415 & 2.0902148433 & 0.0106389366 \\ \mathrm{H} & 1.0238886738 & 2.4598550456 & 0.0590811124 \\ \mathrm{H} & -0.3771726849 & 1.9326883138 & 1.0204946273 \\ \mathrm{C} & 0.8399357787 & -1.5824302661 & -0.8039821805 \\ \mathrm{H} & 1.4641336722 & -1.5153871901 & -1.7047177203 \\ \mathrm{H} & -0.1778893793 & -1.8466227763 & -1.0919686125 \\ \mathrm{H} & -0.6372906022 & 2.7949754554 & -0.5262636261 \\ \mathrm{H} & 1.2630129536 & -2.3606871200 & -0.1651135879 \\ \mathrm{H} & -1.5172088577 & -0.6619547157 & 0.6170341791\end{array}$




$\begin{array}{llll}\mathrm{O} & -2.2572132012 & -0.4208614151 & -0.2923146550 \\ \mathrm{H} & -2.9617769023 & 0.1533147238 & 0.0405383345\end{array}$

\section{TS1 $_{\text {aq }}$}

\begin{tabular}{|c|c|c|c|}
\hline \multicolumn{2}{|c|}{ Solution phase energy: } & \multicolumn{2}{|c|}{-401.50112819478} \\
\hline $\mathrm{C}$ & -0.2414694796 & 0.0041852643 & 0.1833046183 \\
\hline $\mathrm{H}$ & 0.7782169597 & -0.1434815352 & 0.5564474448 \\
\hline $\mathrm{H}$ & -0.4281409379 & 1.0759202523 & 0.0822777652 \\
\hline $\mathrm{C}$ & -0.3767066790 & -0.7117200889 & -1.1412648358 \\
\hline $\mathrm{O}$ & -2.2475105392 & -0.2927642421 & -1.5176070823 \\
\hline $\mathrm{O}$ & -0.3855209288 & -2.0235742187 & -1.1061559316 \\
\hline $\mathrm{H}$ & -1.2057120373 & -2.4215264905 & -1.7795502178 \\
\hline $\mathrm{N}$ & 0.2574095012 & -0.1104297556 & -2.1998307082 \\
\hline $\mathrm{H}$ & 0.1368538473 & 0.8952255919 & -2.2321981918 \\
\hline $\mathrm{C}$ & 0.2955493196 & -0.7537004789 & -3.5085981430 \\
\hline $\mathrm{H}$ & -0.6751972127 & -0.7370849126 & -4.0214462472 \\
\hline $\mathrm{H}$ & 0.6098581825 & -1.7919076543 & -3.3865479433 \\
\hline $\mathrm{H}$ & -2.2049416931 & -2.5571345848 & -3.3695206124 \\
\hline $\mathrm{O}$ & -2.3345840529 & -2.5098978861 & -2.4118967735 \\
\hline $\mathrm{H}$ & -2.4704025137 & -1.3967778996 & -2.0761313147 \\
\hline I & -2.7203626789 & -0.3445298455 & -0.6724276819 \\
\hline $\mathrm{H}$ & 1.0276009870 & -0.2407786290 & -4.1366255860 \\
\hline 1 & -0.9373729534 & -0.4223355988 & 0.9081021981 \\
\hline
\end{tabular}

$\mathbf{2}_{\mathrm{aq}}$

Solution phase energy: $\quad-401.53244847674$

$\begin{array}{llll}\text { C } & 0.0055487354 & 0.0494005914 & -0.0392664518\end{array}$ 


\begin{tabular}{|c|c|c|c|}
\hline $\mathrm{H}$ & 1.0974559506 & 0.0486421357 & -0.0153420401 \\
\hline $\mathrm{H}$ & -0.3523481117 & 1.0822974506 & -0.0616427364 \\
\hline $\mathrm{C}$ & -0.5115089436 & -0.7121077718 & -1.2565178704 \\
\hline $\mathrm{O}$ & -1.9162528591 & -0.6880613012 & -1.1978178099 \\
\hline $\mathrm{H}$ & -2.2385402054 & -1.2171030421 & -1.9537908940 \\
\hline $\mathrm{O}$ & 0.0067701614 & -2.0185035856 & -1.1869158499 \\
\hline $\mathrm{H}$ & -0.3793517519 & -2.5191772748 & -1.9284293450 \\
\hline $\mathrm{N}$ & -0.1341216446 & -0.1311914969 & -2.5703364399 \\
\hline $\mathrm{H}$ & -0.5964726873 & 0.7731033188 & -2.6491669553 \\
\hline $\mathrm{C}$ & 1.3060570879 & 0.0083831315 & -2.8235527256 \\
\hline $\mathrm{H}$ & 1.4591670560 & 0.3203547099 & -3.8606567677 \\
\hline $\mathrm{H}$ & 1.7836775834 & -0.9630902895 & -2.6815242163 \\
\hline $\mathrm{H}$ & -1.0388714488 & -1.3895134702 & -3.5957504451 \\
\hline $\mathrm{O}$ & -1.6072535969 & -2.2007677227 & -3.5726664696 \\
\hline $\mathrm{H}$ & -1.9329080252 & -2.3606465845 & -4.4689779907 \\
\hline $\mathrm{H}$ & 1.8046576588 & 0.7369071092 & -2.1689304472 \\
\hline $\mathrm{H}$ & -0.3622122147 & -0.4304116724 & 0.8712529904 \\
\hline
\end{tabular}

\section{TS2 $_{\text {aq }}$}

\begin{tabular}{lrll}
\multicolumn{2}{l}{ Solution phase energy: } & -401.51350470749 \\
$\mathrm{C}$ & 0.0079324893 & 0.0608156632 & -0.0457870955 \\
$\mathrm{H}$ & 1.0997696667 & 0.0808620843 & -0.0046405735 \\
$\mathrm{H}$ & -0.3810258568 & 1.0824127040 & -0.0397389814 \\
$\mathrm{C}$ & -0.5007598638 & -0.6896098572 & -1.2638242411 \\
$\mathrm{O}$ & -1.8380876172 & -0.7264726825 & -1.3616154117 \\
$\mathrm{H}$ & -1.9654533164 & -1.2972654826 & -2.3670509296 \\
$\mathrm{O}$ & 0.1265140745 & -1.9402603824 & -1.3368242654
\end{tabular}




$\begin{array}{llll}\mathrm{H} & -0.3581613754 & -2.4315850569 & -2.0332521093 \\ \mathrm{~N} & -0.0291414407 & 0.0631512450 & -2.6122666687 \\ \mathrm{H} & -0.4534685656 & 0.9923247205 & -2.6120289768 \\ \mathrm{C} & 1.4206673542 & 0.1283304737 & -2.9029567616 \\ \mathrm{H} & 1.5720319490 & 0.4686073914 & -3.9296983578 \\ \mathrm{H} & 1.8346060546 & -0.8722161805 & -2.7789895967 \\ \mathrm{H} & -0.5683794468 & -0.5384431087 & -3.3159095951 \\ \mathrm{O} & -1.5854628036 & -1.8255981477 & -3.4695394697 \\ \mathrm{H} & -2.3012232707 & -1.9090490401 & -4.1122351638 \\ \mathrm{H} & 1.9116066308 & 0.8183808530 & -2.2142980998 \\ \mathrm{H} & -0.3637554287 & -0.4553693473 & 0.8431653382\end{array}$

$3_{\text {aq }}$

Solution phase energy: $\quad-401.56226984168$

$\begin{array}{lllll}\text { C } & -3.4976769553 & -0.5988472059 & -0.2179666870\end{array}$

$\begin{array}{llll}\text { C } & -2.1288651818 & 0.0310872296 & -0.1112114970\end{array}$

$\begin{array}{llll}\mathrm{O} & -1.4301103720 & -0.4189137560 & 0.9269407118\end{array}$

$\begin{array}{llll}\text { O } & -1.7014628762 & 0.8729918999 & -0.8933626117\end{array}$

$\begin{array}{llll}\text { O } & 0.7840654113 & 0.9245848206 & 0.7286905197\end{array}$

$\mathrm{N} \quad 2.7684336371 \quad-0.6769677288 \quad-0.2961275765$

$\begin{array}{lllll}\text { C } & 4.1013174426 & -0.0585494549 & -0.1837795127\end{array}$

$\mathrm{H} \quad-4.0168251584 \quad-0.2176397110 \quad-1.0975575381$

$\mathrm{H} \quad-3.4034453090 \quad-1.6876862192 \quad-0.2819196240$

$\begin{array}{llll}\mathrm{H} & -4.07886559533 & -0.3736170539 & 0.6826644296\end{array}$

H $\quad 2.5726582838 \quad-0.9180284136 \quad-1.2653471654$

$\begin{array}{llll}\mathrm{H} & 4.3008728778 & 0.1774401223 & 0.8656540071\end{array}$

$\begin{array}{llll}\mathrm{H} & 4.1134199697 & 0.8817847887 & -0.7422172291\end{array}$ 


$\begin{array}{llll}\mathrm{H} & 4.9190038122 & -0.6899897613 & -0.5559020103 \\ \mathrm{H} & 2.7515696171 & -1.5579988190 & 0.2141911651 \\ \mathrm{H} & -0.5298407409 & 0.0597948986 & 0.9326007821 \\ \mathrm{H} & 0.4154803952 & 1.4170080981 & -0.0218785286 \\ \mathrm{H} & 1.5101108177 & 0.3469831795 & 0.3361827335\end{array}$

\section{Methylamine}

\begin{tabular}{|c|c|c|c|}
\hline \multicolumn{2}{|c|}{ Solution phase energy: } & \multicolumn{2}{|c|}{-95.90018930609} \\
\hline $\mathrm{C}$ & -2.5120114607 & -0.1572197312 & 0.0093757975 \\
\hline $\mathrm{H}$ & -2.1689016302 & -1.1972500667 & 0.0175447818 \\
\hline $\mathrm{H}$ & -2.1824441145 & 0.3093472583 & 0.9507551745 \\
\hline $\mathrm{H}$ & -3.6069258136 & -0.1801958798 & 0.0170402326 \\
\hline $\mathrm{N}$ & -2.0433469271 & 0.5058827462 & -1.2142581247 \\
\hline $\mathrm{H}$ & -2.3499126223 & 1.4771703796 & -1.2151998746 \\
\hline $\mathrm{H}$ & -1.0255507112 & 0.5435296420 & -1.2139955377 \\
\hline
\end{tabular}

\section{Acetate}

\begin{tabular}{|c|c|c|c|}
\hline \multicolumn{2}{|c|}{ Solution phase energy: } & \multicolumn{2}{|c|}{-228.69283075472} \\
\hline $\mathrm{C}$ & 0.4892683773 & 1.3522122137 & 0.0000000000 \\
\hline $\mathrm{H}$ & 1.5816760049 & 1.3947087251 & 0.0000000000 \\
\hline $\mathrm{H}$ & 0.1084980085 & 1.8839720125 & -0.8797667641 \\
\hline $\mathrm{H}$ & 0.1084980085 & 1.8839720125 & 0.8797667641 \\
\hline $\mathrm{C}$ & -0.0328005090 & -0.1061479253 & 0.0000000000 \\
\hline $\mathrm{O}$ & -1.2871345361 & -0.2371342054 & 0.0000000000 \\
\hline & 0.8313420996 & -1.0230051958 & 0.000000000 \\
\hline
\end{tabular}




\section{$\mathbf{1 A}$}

Solution phase energy: $\quad-1643.04127337993$

$\begin{array}{lllll}\text { C } & 0.8558710000 & -2.2880560000 & -1.5085620000\end{array}$

$\begin{array}{llll}\text { C } & 0.2964789833 & -3.5543414026 & -0.8694682463\end{array}$

$\begin{array}{lllll}\text { O } & 0.8242310760 & -4.6450179299 & -1.0122624215\end{array}$

$\begin{array}{llll}\text { O } & -0.8096307352 & -3.4042730696 & -0.1482602876\end{array}$

$\begin{array}{llll}\text { C } & 3.0048710000 & -2.6820560000 & 2.2034379999\end{array}$

$\begin{array}{llll}\mathrm{N} & 2.1257701294 & -3.5297515917 & 2.8469081354\end{array}$

$\begin{array}{llll}\text { C } & 2.2620018567 & -1.5756915303 & 1.8761634539\end{array}$

$\begin{array}{lllll}\text { C } & 0.9124248876 & -2.9367397577 & 2.8886074265\end{array}$

$\begin{array}{lllll}\mathrm{N} & 0.9523043497 & -1.7421239006 & 2.3080060279\end{array}$

$\begin{array}{llll}\text { C } & -2.3431290000 & -2.0580559999 & 6.0914380000\end{array}$

$\begin{array}{llll}\text { O } & -1.4003111210 & -2.4467751763 & 5.0992569392\end{array}$

$\begin{array}{llll}\text { C } & -0.1441290000 & 2.6499440000 & 5.0764379999\end{array}$

$\begin{array}{llll}\mathrm{N} & 0.2181382614 & 1.5061255533 & 5.7616648357\end{array}$

$\begin{array}{llll}\text { C } & -0.5214742040 & 2.2319801078 & 3.8333328485\end{array}$

$\begin{array}{llll}\text { C } & 0.0599840868 & 0.4501158031 & 4.9336315220\end{array}$

$\begin{array}{llll}\mathrm{N} & -0.3875727423 & 0.8561624967 & 3.7512978380\end{array}$

$\begin{array}{llll}\text { C } & 0.0438710000 & 2.8819439999 & -0.6085620000\end{array}$

$\begin{array}{lllll}\text { C } & -0.2206926377 & 1.7685035177 & 0.3793112491\end{array}$

$\begin{array}{lllll}\text { O } & 0.6617687284 & 0.8558869968 & 0.5385308322\end{array}$

$\begin{array}{llll}\text { O } & -1.3107656619 & 1.7502312913 & 1.0181993974\end{array}$

$\begin{array}{lllll}\text { C } & -3.3011290000 & 0.1699440000 & -4.2235619998\end{array}$

$\begin{array}{llll}\mathrm{N} & -2.0349557927 & 0.7166940536 & -4.2330964172\end{array}$

$\begin{array}{llll}\text { C } & -3.4693760046 & -0.3813596701 & -2.9835417636\end{array}$

$\begin{array}{lllll}\text { C } & -1.4571031520 & 0.5033482572 & -3.0427976467\end{array}$

$\begin{array}{lllll}\mathrm{N} & -2.3115271217 & -0.1612913228 & -2.2653680509\end{array}$ 


\begin{tabular}{|c|c|c|c|}
\hline $\mathrm{Zn}$ & -0.6760579505 & -0.2516994938 & 1.9308036321 \\
\hline $\mathrm{O}$ & -1.3569658634 & -0.9601522217 & -0.0128514698 \\
\hline $\mathrm{O}$ & -2.3577316769 & -1.3081450339 & 2.7729755136 \\
\hline $\mathrm{C}$ & -3.2953876479 & -3.4920621493 & 2.3603845649 \\
\hline $\mathrm{H}$ & 1.2156444311 & -1.5893368087 & -0.7491901881 \\
\hline $\mathrm{H}$ & 0.0943581033 & -1.7965239419 & -2.1186866072 \\
\hline $\mathrm{H}$ & 2.5677164409 & -0.6746654231 & 1.3663296420 \\
\hline $\mathrm{H}$ & 2.3401111903 & -4.4488941112 & 3.2136493913 \\
\hline $\mathrm{H}$ & 0.0537549839 & -3.3865115465 & 3.3631465580 \\
\hline $\mathrm{H}$ & -2.3228381931 & -0.9754364990 & 6.2803616511 \\
\hline $\mathrm{H}$ & -1.6751086007 & -2.0142799425 & 4.2662564044 \\
\hline $\mathrm{H}$ & 0.2369450683 & 3.8112502208 & -0.0597877047 \\
\hline $\mathrm{H}$ & -0.8417809319 & 3.0585252322 & -1.2261175549 \\
\hline $\mathrm{H}$ & -4.3104872727 & -0.9149706086 & -2.5680626654 \\
\hline $\mathrm{H}$ & -2.0465613492 & -0.5057332140 & -1.2354943730 \\
\hline $\mathrm{H}$ & -0.4591016611 & 0.8164937843 & -2.7768165107 \\
\hline $\mathrm{H}$ & -0.8799265984 & 2.8044190394 & 2.9938735278 \\
\hline $\mathrm{H}$ & 0.2400219246 & -0.5760253452 & 5.2191007720 \\
\hline $\mathrm{H}$ & -0.5044768296 & -0.6067588256 & -0.3132956864 \\
\hline $\mathrm{H}$ & -2.9738595089 & -3.7939115373 & 3.3607021982 \\
\hline $\mathrm{H}$ & -2.5577813442 & -3.8717106562 & 1.6438340082 \\
\hline $\mathrm{H}$ & -4.2694170447 & -3.9333648979 & 2.1366993355 \\
\hline $\mathrm{H}$ & -0.1021674755 & 3.6288013821 & 5.5296583834 \\
\hline $\mathrm{H}$ & 0.5500106299 & 1.4597067333 & 6.7168394637 \\
\hline $\mathrm{H}$ & -3.3710044734 & -2.3407039494 & 5.8235550816 \\
\hline $\mathrm{H}$ & -2.0840872237 & -2.5718956722 & 7.0220876996 \\
\hline $\mathrm{H}$ & 4.0409996665 & -2.9359594800 & 2.0398917303 \\
\hline
\end{tabular}




$\begin{array}{llll}\mathrm{H} & 1.6970750097 & -2.5621411648 & -2.1476589637 \\ \mathrm{H} & -3.9567724881 & 0.2200266317 & -5.0793593038 \\ \mathrm{C} & -3.3045599938 & -1.9878431471 & 2.2787268272 \\ \mathrm{H} & -1.0847343773 & -2.4005676905 & -0.0963982303 \\ \mathrm{~N} & -4.3313574236 & -1.3957654078 & 1.6761593693 \\ \mathrm{H} & -5.0840998969 & -1.9806684084 & 1.3368428717 \\ \mathrm{C} & -4.4479827459 & 0.0535199710 & 1.5253839484 \\ \mathrm{H} & -5.3265087612 & 0.2657542743 & 0.9142528906 \\ \mathrm{H} & -4.5701774578 & 0.5366982150 & 2.5000263166 \\ \mathrm{H} & -3.5567604107 & 0.4591717154 & 1.0423842523 \\ \mathrm{H} & 0.9109821798 & 2.6670175765 & -1.2353285227 \\ \mathrm{H} & -1.6022970632 & 1.2040047589 & -5.0100810873\end{array}$

\section{B}

Solution phase energy: $\quad-1643.00712149654$

$\begin{array}{llll}\mathrm{C} & 0.0000000004 & -0.0000000002 & -0.0000000004 \\ \mathrm{C} & 0.2255408452 & 0.2726585495 & 1.5073384267 \\ \mathrm{O} & -0.3740219174 & 1.2555100270 & 2.0034831644 \\ \mathrm{O} & 0.9866055710 & -0.5384363945 & 2.1205927317 \\ \mathrm{C} & -2.5764000003 & -1.1808000001 & 3.2435000005 \\ \mathrm{~N} & -1.4144277504 & -1.7249252141 & 3.7442889057 \\ \mathrm{C} & -2.7964542051 & -1.8133104348 & 2.0522087521 \\ \mathrm{C} & -0.9518040349 & -2.6254715427 & 2.8570771454 \\ \mathrm{~N} & -1.7737684626 & -2.7231170067 & 1.8204862378 \\ \mathrm{C} & -0.8189000002 & -7.5843999992 & 3.1388000000 \\ \mathrm{O} & -1.8949593016 & -7.3700557702 & 2.2157751993 \\ \mathrm{C} & -4.9297010006 & -6.6663000003 & -0.0689000011\end{array}$




$\begin{array}{llll}\mathrm{N} & -5.2563436560 & -5.9788729384 & 1.0820741818 \\ \mathrm{C} & -3.8263492766 & -6.0354076990 & -0.5742358496 \\ \mathrm{C} & -4.3592336137 & -4.9813562020 & 1.2441510448 \\ \mathrm{~N} & -3.4756594280 & -4.9868508463 & 0.2552622254 \\ \mathrm{C} & -3.0900999994 & -2.5191000004 & -3.5076999982 \\ \mathrm{C} & -2.4184356567 & -2.9467375567 & -2.2169115559 \\ \mathrm{O} & -2.5622676265 & -2.2382431104 & -1.1722656731 \\ \mathrm{O} & -1.7536114808 & -4.0334364349 & -2.1692433299 \\ \mathrm{C} & 2.2397999999 & -1.3682999996 & -4.8790000009 \\ \mathrm{~N} & 1.1848408267 & -1.0811939512 & -4.0355220748 \\ \mathrm{C} & 2.9332066445 & -2.3907599537 & -4.3067942852 \\ \mathrm{C} & 1.2166034464 & -1.8945025506 & -2.9780388110 \\ \mathrm{~N} & 2.2768866504 & -2.6957943462 & -3.1302068732 \\ \mathrm{Zn} & -1.6401048286 & -3.8169335668 & 0.0107402519 \\ \mathrm{O} & 0.6645658635 & -3.4488029354 & -0.3234811470 \\ \mathrm{O} & -0.6831292726 & -5.6248958028 & 0.6599591355 \\ \mathrm{C} & 2.7208915031 & -2.6937681287 & 0.7040273180 \\ \mathrm{H} & -0.3838946979 & 0.8917452784 & -0.5045716800 \\ \mathrm{H} & -0.7294113713 & -0.8052423971 & -0.1261303428 \\ \mathrm{H} & -3.5872159091 & -1.6594627818 & 1.3341583664 \\ \mathrm{H} & -0.0232446388 & -3.1564365938 & 2.9871701900 \\ \mathrm{H} & -1.0610626434 & -8.3805062271 & 3.8508297610 \\ \mathrm{H} & -2.0776153226 & -8.2006806725 & 1.7513311055 \\ \mathrm{H} & -4.0569513362 & -3.0320427569 & -3.5791405817 \\ \mathrm{H} & -2.4961530231 & -2.8152481461 & -4.3760719217 \\ \mathrm{H} & 3.8195948394 & -2.9157724378 & -4.6282544120 \\ \mathrm{H} & 2.5636545238 & -3.4054939948 & -2.4552587109 \\ \mathrm{H} & & & \\ \mathrm{H} & & & \end{array}$




\begin{tabular}{|c|c|c|c|}
\hline $\mathrm{H}$ & 0.5318587014 & -1.9084222469 & -2.1438079587 \\
\hline $\mathrm{H}$ & -3.2706833533 & -6.2474385071 & -1.4748653017 \\
\hline $\mathrm{H}$ & -4.3787726065 & -4.2887855142 & 2.0729889467 \\
\hline $\mathrm{H}$ & -1.2367366786 & -6.2523532024 & 1.1992993562 \\
\hline $\mathrm{H}$ & 2.0719753279 & -2.0088139495 & 1.2701915955 \\
\hline $\mathrm{H}$ & 3.3453406067 & -2.0887082310 & 0.0342223130 \\
\hline $\mathrm{H}$ & 3.3987838465 & -3.2444685339 & 1.3657024740 \\
\hline $\mathrm{H}$ & 0.5018809575 & -0.3453716110 & -4.1828356684 \\
\hline $\mathrm{H}$ & -6.0340907462 & -6.1779622849 & 1.6987797774 \\
\hline $\mathrm{H}$ & 0.9377982381 & -0.3125020540 & -0.4730216069 \\
\hline $\mathrm{H}$ & 2.4030296480 & -0.8264561683 & -5.7979990523 \\
\hline $\mathrm{H}$ & 0.1165714872 & -7.8411827409 & 2.6251753667 \\
\hline $\mathrm{H}$ & -0.6828675917 & -6.6527011288 & 3.6922976463 \\
\hline $\mathrm{H}$ & -3.1185884518 & -0.4038722608 & 3.7598370577 \\
\hline $\mathrm{H}$ & -0.9163918847 & -1.4105891123 & 4.5663282586 \\
\hline $\mathrm{H}$ & -5.5085534524 & -7.5076115100 & -0.4197043678 \\
\hline $\mathrm{C}$ & 1.8765747600 & -3.6355796077 & -0.1173150684 \\
\hline $\mathrm{C}$ & 1.8497307462 & -5.7616781920 & -1.4233154984 \\
\hline $\mathrm{H}$ & 1.5209802132 & -6.5454719913 & -0.7336098555 \\
\hline $\mathrm{H}$ & 0.9684417505 & -5.3645041023 & -1.9276799751 \\
\hline $\mathrm{H}$ & 2.5329716529 & -6.1982012958 & -2.1554079035 \\
\hline $\mathrm{N}$ & 2.5341344327 & -4.6715589769 & -0.7215774697 \\
\hline $\mathrm{H}$ & 3.4866407122 & -4.8443406705 & -0.4199443745 \\
\hline $\mathrm{H}$ & 0.1574912013 & -5.5131612665 & 1.1193124245 \\
\hline $\mathrm{H}$ & -3.2769224459 & -1.4440937123 & -3.5165778888 \\
\hline
\end{tabular}




\section{B (Alternative $\mathrm{OH}^{-}$and Glu- $\mathrm{H}^{+}$)}

Solution phase energy: $\quad-1643.02975787374$

$\begin{array}{llll}\text { C } & -0.0000001619 & 0.0000001951 & 0.0000001898\end{array}$

$\begin{array}{llll}\text { C } & 0.5548281621 & 0.0151389810 & 1.4067544779\end{array}$

$\begin{array}{llll}\text { O } & 0.1345071978 & 0.7368848638 & 2.2901691139\end{array}$

$\begin{array}{llll}\text { O } & 1.5663948630 & -0.8302975619 & 1.6617196102\end{array}$

C $\quad-2.5764002025 \quad-1.1807996107 \quad 3.2435004608$

$\mathrm{N} \quad-1.7380087342 \quad-2.0713740845 \quad 3.8795765139$

C $\quad-2.6557658563 \quad-1.6148502650 \quad 1.9466700315$

C $\quad-1.3348240043 \quad-2.9930943600 \quad 2.9772214235$

$\begin{array}{llll}\mathrm{N} & -1.8763788789 & -2.7479837893 & 1.7898008910\end{array}$

$\begin{array}{llll}\text { C } & -0.8189000135 & -7.5843999905 & 3.1388000021\end{array}$

$\begin{array}{llll}\text { O } & -1.0384753819 & -6.2232400962 & 2.7626506499\end{array}$

$\begin{array}{llll}\text { C } & -4.9297005798 & -6.6663004125 & -0.0689000332\end{array}$

$\mathrm{N} \quad \quad-5.3100326874 \quad-5.8679413492 \quad 0.9933389130$

C $\quad-3.6749665996 \quad-6.2520163000 \quad-0.4121091902$

C $\quad-4.3012821239 \quad-5.0097344911 \quad 1.2561826156$

$\mathrm{N} \quad-3.2892873834 \quad-5.2134285116 \quad 0.4182940934$

C $\quad-3.0901002395 \quad-2.5190999304 \quad-3.5077002020$

C $\quad-2.3868620193 \quad-3.1573807567 \quad-2.3212882609$

O $\quad-1.9562660759 \quad-2.3945317532 \quad-1.3726672073$

O $\quad-2.2644426711 \quad-4.4014925363 \quad-2.2617177908$

C $\quad 2.2397999438 \quad-1.3683001356 \quad-4.8789996068$

$\mathrm{N} \quad 0.9160776509 \quad-1.3224180834 \quad-4.4829055849$

C $\quad 2.9514852204 \quad-1.7581861208 \quad-3.7811034780$

C $\quad 0.8172867550 \quad-1.6747513724 \quad-3.1941063875$

$\mathrm{N} \quad 2.0468501962 \quad-1.9421503941 \quad-2.7526250999$ 


\begin{tabular}{|c|c|c|c|}
\hline $\mathrm{Zn}$ & -1.5376265110 & -3.9710610953 & -0.0143156666 \\
\hline $\mathrm{O}$ & 2.6950549276 & -2.4629824459 & -0.1887439698 \\
\hline $\mathrm{O}$ & 0.1410268249 & -4.8697552780 & 0.1873205428 \\
\hline $\mathrm{C}$ & 3.8440939293 & -3.0267280682 & 1.8513699150 \\
\hline $\mathrm{H}$ & -0.7019901597 & 0.8263464376 & -0.1193409188 \\
\hline $\mathrm{H}$ & -0.5263443790 & -0.9381196821 & -0.1966166828 \\
\hline $\mathrm{H}$ & -3.1980094611 & -1.1864091998 & 1.1167581980 \\
\hline $\mathrm{H}$ & -0.6969001341 & -3.8313063916 & 3.2157113810 \\
\hline $\mathrm{H}$ & -1.7257146714 & -8.0503178556 & 3.5469586596 \\
\hline $\mathrm{H}$ & -1.7132058463 & -6.1999477708 & 2.0685910555 \\
\hline $\mathrm{H}$ & -4.1636342540 & -2.7246891601 & -3.4270772386 \\
\hline $\mathrm{H}$ & -2.7465119723 & -2.9748190643 & -4.4407248233 \\
\hline $\mathrm{H}$ & 4.0119409774 & -1.9091490876 & -3.6483683293 \\
\hline $\mathrm{H}$ & 2.2881906583 & -2.2369818309 & -1.7705141574 \\
\hline $\mathrm{H}$ & -0.0958089244 & -1.7386977992 & -2.6136281398 \\
\hline $\mathrm{H}$ & -3.0367195476 & -6.6008270642 & -1.2076402429 \\
\hline $\mathrm{H}$ & -4.3383895830 & -4.2609687964 & 2.0333404749 \\
\hline $\mathrm{H}$ & 0.1379801238 & -5.2956672053 & 1.0572019931 \\
\hline $\mathrm{H}$ & 2.9819559719 & -3.0215471856 & 2.5257578446 \\
\hline $\mathrm{H}$ & 4.2919576288 & -2.0301350371 & 1.8842653891 \\
\hline $\mathrm{H}$ & 4.5658036909 & -3.7650352384 & 2.2083491823 \\
\hline $\mathrm{H}$ & 0.1355131022 & -1.0562540208 & -5.0726782242 \\
\hline $\mathrm{H}$ & -6.1914227150 & -5.9107777508 & 1.4899268075 \\
\hline $\mathrm{H}$ & 0.8104280926 & 0.1056906518 & -0.7294476290 \\
\hline $\mathrm{H}$ & 2.5503024193 & -1.1204402639 & -5.8822300882 \\
\hline $\mathrm{H}$ & -0.4579321498 & -8.1922705581 & 2.2989162715 \\
\hline $\mathrm{H}$ & -0.0527489895 & -7.5840193058 & 3.9184214919 \\
\hline
\end{tabular}




$\begin{array}{llll}\mathrm{H} & -3.0133022681 & -0.3355751638 & 3.7529945920 \\ \mathrm{H} & -1.4481177894 & -2.0301875182 & 4.8485379891 \\ \mathrm{H} & -5.5720033399 & -7.4377021328 & -0.4660552484 \\ \mathrm{C} & 3.3655668891 & -3.3215961293 & 0.4503383136 \\ \mathrm{C} & 3.1149470908 & -5.0152679519 & -1.3120869710 \\ \mathrm{H} & 2.0233543632 & -4.9614599113 & -1.2393101621 \\ \mathrm{H} & 3.4765281861 & -4.4550775638 & -2.1802872527 \\ \mathrm{H} & 3.4206440733 & -6.0562843550 & -1.4261975618 \\ \mathrm{~N} & 3.6921789694 & -4.5017448447 & -0.0727018116 \\ \mathrm{H} & 4.2288812669 & -5.1373720976 & 0.5022613202 \\ \mathrm{H} & 1.8582117968 & -1.3636670201 & 0.8803830165 \\ \mathrm{H} & -2.9465753372 & -1.4374089505 & -3.5327354807\end{array}$

\section{TS1}

\begin{tabular}{lrll}
\multicolumn{3}{l}{ Solution phase energy: } & -1643.00787792381 \\
$\mathrm{C}$ & 0.1944978414 & 0.1939805804 & 0.0531128353 \\
$\mathrm{C}$ & 0.5882881657 & -0.1408385262 & 1.4763494703 \\
$\mathrm{O}$ & 0.4713338642 & 0.6300830065 & 2.4077067885 \\
$\mathrm{O}$ & 1.1086328302 & -1.3636065110 & 1.6812682690 \\
$\mathrm{C}$ & -2.5406118783 & -1.1964005625 & 3.1542778425 \\
$\mathrm{~N}$ & -1.7059005636 & -2.0908064401 & 3.7902323233 \\
$\mathrm{C}$ & -2.6804914259 & -1.6689289324 & 1.8772601680 \\
$\mathrm{C}$ & -1.3614911052 & -3.0524458093 & 2.9081663279 \\
$\mathrm{~N}$ & -1.9432139739 & -2.8326097989 & 1.7343955837 \\
$\mathrm{C}$ & -0.6843129134 & -7.5797663003 & 3.1082429566 \\
$\mathrm{O}$ & -0.7695398735 & -6.1849029391 & 2.8390436117 \\
$\mathrm{C}$ & -5.0623017613 & -6.6849253072 & 0.0012710602
\end{tabular}




\begin{tabular}{|c|c|c|c|}
\hline $\mathrm{N}$ & -4.7584845037 & -6.6021112650 & 1.3456132638 \\
\hline $\mathrm{C}$ & -4.1370569451 & -5.9036377771 & -0.6348391385 \\
\hline $\mathrm{C}$ & -3.6871471831 & -5.7941313367 & 1.4898259342 \\
\hline $\mathrm{N}$ & -3.2827636181 & -5.3486469799 & 0.3044973132 \\
\hline $\mathrm{C}$ & -3.0048872128 & -2.5572143499 & -3.5958832891 \\
\hline $\mathrm{C}$ & -2.3122171278 & -3.1626211156 & -2.3906855017 \\
\hline $\mathrm{O}$ & -1.9733215490 & -2.3965530217 & -1.4117072106 \\
\hline $\mathrm{O}$ & -2.1100605243 & -4.4024311524 & -2.3355495589 \\
\hline $\mathrm{C}$ & 2.1769743845 & -1.3754940323 & -4.8152928308 \\
\hline $\mathrm{N}$ & 0.9264994514 & -0.9201160289 & -4.4445414832 \\
\hline $\mathrm{C}$ & 2.5886309162 & -2.2156417240 & -3.8206056296 \\
\hline $\mathrm{C}$ & 0.5874454629 & -1.4600930029 & -3.2658993416 \\
\hline $\mathrm{N}$ & 1.5851990915 & -2.2512905041 & -2.8740743431 \\
\hline $\mathrm{Zn}$ & -1.6621505591 & -4.0061721982 & -0.0575331512 \\
\hline $\mathrm{O}$ & 1.6282163963 & -3.1501524982 & -0.3551120368 \\
\hline $\mathrm{O}$ & 0.0927853929 & -4.8156488439 & 0.3613841954 \\
\hline $\mathrm{C}$ & 2.3761761439 & -5.0765063223 & 0.9993459222 \\
\hline $\mathrm{H}$ & -0.0970619461 & 1.2435068400 & -0.0033130803 \\
\hline $\mathrm{H}$ & -0.6406135882 & -0.4373630800 & -0.2696524421 \\
\hline $\mathrm{H}$ & -3.2492543763 & -1.2554819009 & 1.0572662679 \\
\hline $\mathrm{H}$ & -0.7293646540 & -3.8936956532 & 3.1490560695 \\
\hline $\mathrm{H}$ & -1.2574604658 & -8.1804075759 & 2.3876470464 \\
\hline $\mathrm{H}$ & -0.3645169578 & -5.9982627889 & 1.9743487597 \\
\hline $\mathrm{H}$ & -4.0843433876 & -2.7189505169 & -3.4892031756 \\
\hline $\mathrm{H}$ & -2.6868273433 & -3.0575673519 & -4.5139702304 \\
\hline $\mathrm{H}$ & 3.5000472240 & -2.7838495684 & -3.7148004293 \\
\hline $\mathrm{H}$ & 1.6079152628 & -2.7582752148 & -1.9389354810 \\
\hline
\end{tabular}




\begin{tabular}{|c|c|c|c|}
\hline $\mathrm{H}$ & -0.3325842983 & -1.2985745101 & -2.7218180744 \\
\hline $\mathrm{H}$ & -4.0072226022 & -5.7019503198 & -1.6862806639 \\
\hline $\mathrm{H}$ & -3.2090398474 & -5.5854996361 & 2.4343141997 \\
\hline $\mathrm{H}$ & 2.2526500669 & -6.1311953563 & 1.2670486393 \\
\hline $\mathrm{H}$ & 2.2242241360 & -4.4708491697 & 1.8965516826 \\
\hline $\mathrm{H}$ & 3.4032829706 & -4.9389700782 & 0.6413151336 \\
\hline $\mathrm{H}$ & 0.3518170810 & -0.2743472295 & -4.9747834973 \\
\hline $\mathrm{H}$ & -5.2492029411 & -7.0667533125 & 2.0993901001 \\
\hline $\mathrm{H}$ & 1.0373393594 & 0.0148851557 & -0.6230539239 \\
\hline $\mathrm{H}$ & 2.6522576683 & -1.0704452346 & -5.7348947565 \\
\hline $\mathrm{H}$ & 0.3531738781 & -7.9424438535 & 3.1135879283 \\
\hline $\mathrm{H}$ & -1.1069229197 & -7.7484448624 & 4.1034898332 \\
\hline $\mathrm{H}$ & -2.9354036028 & -0.3223707867 & 3.6495873224 \\
\hline $\mathrm{H}$ & -1.3640676561 & -2.0169272282 & 4.7401328911 \\
\hline $\mathrm{H}$ & -5.8865411621 & -7.2753000339 & -0.3694293602 \\
\hline $\mathrm{C}$ & 1.3363695545 & -4.7172908385 & -0.0719715301 \\
\hline $\mathrm{C}$ & 0.6770055415 & -6.4056502595 & -1.7738389334 \\
\hline $\mathrm{H}$ & -0.3044944331 & -5.9879871345 & -1.9973902054 \\
\hline $\mathrm{H}$ & 1.0744631389 & -6.8453220416 & -2.6942983736 \\
\hline $\mathrm{H}$ & 0.5515935352 & -7.2136888422 & -1.0366215927 \\
\hline $\mathrm{N}$ & 1.5962285979 & -5.3476307114 & -1.3466563664 \\
\hline $\mathrm{H}$ & 2.5554560421 & -5.6786769591 & -1.3839939276 \\
\hline $\mathrm{H}$ & 1.1125850566 & -1.9335440120 & 0.8746324320 \\
\hline $\mathrm{H}$ & -2.8342534199 & -1.4811364218 & -3.6673169218 \\
\hline $\mathrm{H}$ & 2.5617463091 & -3.0050402671 & -0.125801255 \\
\hline
\end{tabular}


Solution phase energy: $\quad-1643.01578849974$

$\begin{array}{llll}\text { C } & 0.0000000000 & 0.0000000000 & 0.0000000000\end{array}$

$\begin{array}{llll}\text { C } & 0.3815590000 & -0.0290790000 & 1.4660400000\end{array}$

$\begin{array}{llll}\text { O } & 0.2473310000 & 0.9203110000 & 2.2135070000\end{array}$

$\begin{array}{llll}\text { O } & 0.9092270000 & -1.1741290000 & 1.9272040000\end{array}$

$\begin{array}{llll}\text { C } & -2.5764000000 & -1.1808000000 & 3.2435000000\end{array}$

$\begin{array}{llll}\mathrm{N} & -1.8394520000 & -2.1288670000 & 3.9220940000\end{array}$

$\begin{array}{llll}\text { C } & -2.6271160000 & -1.6192400000 & 1.9463380000\end{array}$

$\begin{array}{llll}\text { C } & -1.4634720000 & -3.0860800000 & 3.0470270000\end{array}$

$\begin{array}{llll}\mathrm{N} & -1.9292820000 & -2.8113240000 & 1.8324360000\end{array}$

$\begin{array}{llll}\text { C } & -0.8189000000 & -7.5844000000 & 3.1388000000\end{array}$

$\begin{array}{llll}\text { O } & -0.6682170000 & -6.1940380000 & 2.8533090000\end{array}$

$\begin{array}{lllll}\text { C } & -4.9297000000 & -6.6663000000 & -0.0689000000\end{array}$

$\mathrm{N} \quad-4.6799300000 \quad-6.6077260000 \quad 1.2880530000$

$\begin{array}{llll}\text { C } & -3.9727620000 & -5.8901850000 & -0.6567390000\end{array}$

$\begin{array}{llll}\text { C } & -3.6061840000 & -5.8142330000 & 1.4857590000\end{array}$

$\begin{array}{llll}\mathrm{N} & -3.1515670000 & -5.3593480000 & 0.3237480000\end{array}$

$\begin{array}{llll}\text { C } & -3.0901000000 & -2.5191000000 & -3.5077000000\end{array}$

$\begin{array}{llll}\text { C } & -2.3456870000 & -3.1305460000 & -2.3338700000\end{array}$

$\begin{array}{llll}\text { O } & -2.1101490000 & -2.3947360000 & -1.2998240000\end{array}$

$\begin{array}{llll}\mathrm{O} & -2.0169060000 & -4.3416420000 & -2.3518990000\end{array}$

$\begin{array}{llll}\text { C } & 2.2398000000 & -1.3683000000 & -4.8790000000\end{array}$

N $\quad 1.1414910000 \quad-0.8687240000 \quad-4.2057280000$

$\begin{array}{llll}\text { C } & 2.5588940000 & -2.5434040000 & -4.2537720000\end{array}$

$\begin{array}{llll}\text { C } & 0.8094460000 & -1.7056660000 & -3.2099750000\end{array}$

$\begin{array}{llll}\mathrm{N} & 1.6596480000 & -2.7303490000 & -3.2203690000\end{array}$ 


$\begin{array}{llll}\mathrm{Zn} & -1.5967640000 & -3.9550220000 & 0.0083750000 \\ \mathrm{O} & 1.4492530000 & -3.0035710000 & 0.0134300000 \\ \mathrm{O} & 0.1338020000 & -4.8666400000 & 0.2698210000 \\ \mathrm{C} & 2.4078430000 & -5.0498140000 & 0.9515610000 \\ \mathrm{H} & -0.3741940000 & 0.9938700000 & -0.2497990000 \\ \mathrm{H} & -0.7665130000 & -0.7466210000 & -0.2247200000 \\ \mathrm{H} & -3.1087770000 & -1.1654830000 & 1.0927940000 \\ \mathrm{H} & -0.8911520000 & -3.9628600000 & 3.3168580000 \\ \mathrm{H} & -1.5509970000 & -8.0683430000 & 2.4771630000 \\ \mathrm{H} & -0.3137890000 & -6.0570930000 & 1.9545010000 \\ \mathrm{H} & -4.1589360000 & -2.7309040000 & -3.3827600000 \\ \mathrm{H} & -2.7670210000 & -2.9732020000 & -4.4472950000 \\ \mathrm{H} & 3.3472340000 & -3.2510220000 & -4.4625390000 \\ \mathrm{H} & 1.6496800000 & -3.5683510000 & -2.4927710000 \\ \mathrm{H} & -0.0115090000 & -1.5746200000 & -2.5197270000 \\ \mathrm{H} & -3.8002650000 & -5.6760830000 & -1.6990890000 \\ \mathrm{H} & -3.1738210000 & -5.6087630000 & 2.4527320000 \\ \mathrm{H} & 2.4262210000 & -6.1416840000 & 0.8735060000 \\ \mathrm{H} & 2.1214730000 & -4.7811210000 & 1.9713470000 \\ \mathrm{H} & 3.4195250000 & -4.6738450000 & 0.7569420000 \\ \mathrm{H} & 0.6583510000 & -0.0047440000 & -4.4236610000 \\ \mathrm{H} & -5.2089140000 & -7.0734870000 & 2.0148000000 \\ \mathrm{H} & 0.8785030000 & -0.2223820000 & -0.6152650000 \\ \mathrm{H} & 2.6825260000 & -0.8586810000 & -5.7209380000 \\ & 0.1319380000 & -8.1297020000 & 3.0622670000 \\ \mathrm{H} & -1.1777700000 & -7.6680870000 & 4.1687960000 \\ \mathrm{H} & -2.9800970000 & -0.3053640000 & 3.7285260000\end{array}$




$\begin{array}{llll}\mathrm{H} & -1.5889270000 & -2.1008400000 & 4.9027720000 \\ \mathrm{H} & -5.7435500000 & -7.2452910000 & -0.4785740000 \\ \mathrm{C} & 1.3724000000 & -4.4791220000 & -0.0307080000 \\ \mathrm{C} & 1.0960450000 & -6.0081510000 & -2.0211160000 \\ \mathrm{H} & 0.0340530000 & -5.7983930000 & -2.1409890000 \\ \mathrm{H} & 1.5297610000 & -6.2182640000 & -3.0038250000 \\ \mathrm{H} & 1.2142230000 & -6.8969160000 & -1.3892790000 \\ \mathrm{~N} & 1.7680570000 & -4.8161030000 & -1.4632120000 \\ \mathrm{H} & 2.7719780000 & -5.0029780000 & -1.4600940000 \\ \mathrm{H} & 0.9772430000 & -1.8790360000 & 1.2361100000 \\ \mathrm{H} & -2.9673360000 & -1.4353470000 & -3.5419030000 \\ \mathrm{H} & 2.3730060000 & -2.7415070000 & -0.1285280000\end{array}$

\section{TS2}

\begin{tabular}{|c|c|c|c|}
\hline \multicolumn{2}{|c|}{ Solution phase energy: } & \multicolumn{2}{|c|}{-1643.01484149755} \\
\hline $\mathrm{C}$ & 0.0000000000 & 0.0000000001 & 0.0000000001 \\
\hline $\mathrm{C}$ & 0.3450404465 & -0.0362141012 & 1.4732283187 \\
\hline & 0.1515366266 & 0.8899791982 & 2.2353915906 \\
\hline & 0.9038600706 & -1.1720784916 & 1.9280731918 \\
\hline & -2.5764000000 & -1.1808000000 & 3.2435000000 \\
\hline & -1.8233119539 & -2.1187783158 & 3.9187706863 \\
\hline 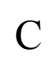 & -2.6418584521 & -1.6295906851 & 1.9508369726 \\
\hline $\mathrm{C}$ & -1.4519708997 & -3.0795193342 & 3.0455862356 \\
\hline & -1.9359483137 & -2.8171597645 & 1.8354256907 \\
\hline & -0.8189000000 & -7.5843999999 & 3.1388000000 \\
\hline & -0.7172816432 & -6.1968113730 & 2.8176818898 \\
\hline & -4.9297000000 & -6.6663000000 & -0.0689000000 \\
\hline
\end{tabular}




$\begin{array}{llll}\mathrm{N} & -4.6895726221 & -6.5924521160 & 1.2892648748 \\ \mathrm{C} & -3.9710475249 & -5.8942239093 & -0.6588537338 \\ \mathrm{C} & -3.6211036555 & -5.7924246262 & 1.4858845601 \\ \mathrm{~N} & -3.1599477254 & -5.3478827547 & 0.3220049686 \\ \mathrm{C} & -3.0901000000 & -2.5191000000 & -3.5076999999 \\ \mathrm{C} & -2.3489650961 & -3.1243647692 & -2.3293226394 \\ \mathrm{O} & -2.0920308139 & -2.3793302554 & -1.3081104015 \\ \mathrm{O} & -2.0413185638 & -4.3422932199 & -2.3287953767 \\ \mathrm{C} & 2.2398000000 & -1.3683000000 & -4.8789999999 \\ \mathrm{~N} & 1.1208370083 & -0.8995962482 & -4.2185880874 \\ \mathrm{C} & 2.5841859580 & -2.5321591123 & -4.2451502551 \\ \mathrm{C} & 0.8079754934 & -1.7495857755 & -3.2240355618 \\ \mathrm{~N} & 1.6835412756 & -2.7510923875 & -3.2186167088 \\ \mathrm{Zn} & -1.5956778671 & -3.9501154446 & 0.0054559028 \\ \mathrm{O} & 1.5253071793 & -2.9959259681 & -0.0171493676 \\ \mathrm{O} & 0.1656720638 & -4.8227672211 & 0.2392285742 \\ \mathrm{C} & 2.4477939332 & -5.0837507473 & 0.8590601029 \\ \mathrm{H} & -0.3210402778 & 1.0079863981 & -0.2670756522 \\ \mathrm{H} & -0.8015958214 & -0.7079840843 & -0.2298976192 \\ \mathrm{H} & -3.1385320858 & -1.1855261369 & 1.1008131969 \\ \mathrm{H} & -0.8705420814 & -3.9502640002 & 3.3141901632 \\ \mathrm{H} & -1.4769373866 & -8.1254895558 & 2.4447439897 \\ \mathrm{H} & -0.3136293026 & -6.0731340078 & 1.9395773688 \\ \mathrm{H} & -4.1663789891 & -2.6420676155 & -3.3351319402 \\ \mathrm{H} & -2.8350794879 & -3.0403370596 & -4.4327892030 \\ \mathrm{H} & 3.3975489253 & -3.2131537362 & -4.4483888774 \\ \mathrm{H} & 1.6849737955 & -3.6525333828 & -2.4362128318 \\ \mathrm{H} & & & \\ \mathrm{H} & & & \end{array}$




\begin{tabular}{|c|c|c|c|}
\hline $\mathrm{H}$ & -0.0256930063 & -1.6367476266 & -2.5459009913 \\
\hline $\mathrm{H}$ & -3.7942318298 & -5.6887274711 & -1.7020442280 \\
\hline $\mathrm{H}$ & -3.1938729026 & -5.5784491231 & 2.4528921614 \\
\hline $\mathrm{H}$ & 2.4211376934 & -6.1740777209 & 0.7659440043 \\
\hline $\mathrm{H}$ & 2.2027966370 & -4.8172361366 & 1.8902750803 \\
\hline $\mathrm{H}$ & 3.4669582723 & -4.7439092699 & 0.6384786458 \\
\hline $\mathrm{H}$ & 0.6141785820 & -0.0507056416 & -4.4422744548 \\
\hline $\mathrm{H}$ & -5.2209735499 & -7.0531629217 & 2.0174659198 \\
\hline $\mathrm{H}$ & 0.8731187695 & -0.2776540341 & -0.5989138007 \\
\hline $\mathrm{H}$ & 2.6765772749 & -0.8476810746 & -5.7174151713 \\
\hline $\mathrm{H}$ & 0.1612418889 & -8.0809101622 & 3.1485565917 \\
\hline $\mathrm{H}$ & -1.2460143049 & -7.6542037322 & 4.1433120748 \\
\hline $\mathrm{H}$ & -2.9766492082 & -0.3027208923 & 3.7265425911 \\
\hline $\mathrm{H}$ & -1.5542480049 & -2.0783231973 & 4.8940485014 \\
\hline $\mathrm{H}$ & -5.7391483603 & -7.2516752853 & -0.4781307726 \\
\hline $\mathrm{C}$ & 1.4033216737 & -4.4577137109 & -0.0784930193 \\
\hline $\mathrm{C}$ & 1.0372193210 & -5.9348952150 & -2.1165482115 \\
\hline $\mathrm{H}$ & -0.0136568108 & -5.6703447364 & -2.2246432859 \\
\hline $\mathrm{H}$ & 1.4572532575 & -6.1474670346 & -3.1043474412 \\
\hline $\mathrm{H}$ & 1.1205804973 & -6.8337358699 & -1.4964106658 \\
\hline $\mathrm{N}$ & 1.7711091896 & -4.7866052000 & -1.5382149356 \\
\hline $\mathrm{H}$ & 2.7651471975 & -5.0229516070 & -1.5470282704 \\
\hline $\mathrm{H}$ & 0.9973827923 & -1.8594070199 & 1.2266723260 \\
\hline $\mathrm{H}$ & -2.8894300748 & -1.4497221198 & -3.6013905589 \\
\hline $\mathrm{H}$ & 2.4640007851 & -2.7580449904 & -0.0826601288 \\
\hline
\end{tabular}


Solution phase energy: $\quad-1643.02688339637$

$\begin{array}{llll}\text { C } & 0.0000000000 & -0.0000000001 & 0.0000000003\end{array}$

$\begin{array}{llll}\text { C } & 0.3299925658 & 0.0495828725 & 1.4747816245\end{array}$

$\begin{array}{llll}\text { O } & 0.1945809195 & 1.0347489441 & 2.1710775425\end{array}$

$\begin{array}{llll}\text { O } & 0.8095044740 & -1.0876988871 & 2.0176477197\end{array}$

$\begin{array}{llll}\text { C } & -2.5764000000 & -1.1808000001 & 3.2434999999\end{array}$

$\begin{array}{llll}\mathrm{N} & -1.8611054896 & -2.1362433102 & 3.9336684808\end{array}$

$\begin{array}{llll}\text { C } & -2.6166549940 & -1.6180704410 & 1.9471473789\end{array}$

$\begin{array}{llll}\text { C } & -1.4840622107 & -3.0979320484 & 3.0654915355\end{array}$

$\begin{array}{llll}\mathrm{N} & -1.9293956064 & -2.8163595825 & 1.8446235166\end{array}$

$\begin{array}{lllll}\text { C } & -0.8189000000 & -7.5843999998 & 3.1387999999\end{array}$

$\begin{array}{llll}\mathrm{O} & -0.6778471858 & -6.1868519960 & 2.8893139410\end{array}$

$\begin{array}{llll}\text { C } & -4.9297000000 & -6.6663000000 & -0.0688999999\end{array}$

$\begin{array}{llll}\mathrm{N} & -4.6770932695 & -6.6351239664 & 1.2878706167\end{array}$

$\begin{array}{llll}\text { C } & -3.9744719830 & -5.8817590238 & -0.6457723066\end{array}$

$\begin{array}{llll}\text { C } & -3.6032343695 & -5.8465988485 & 1.4986846574\end{array}$

$\begin{array}{llll}\mathrm{N} & -3.1520349943 & -5.3710524419 & 0.3432244898\end{array}$

$\begin{array}{llll}\text { C } & -3.0901000001 & -2.51909999999 & -3.5077000002\end{array}$

$\begin{array}{llll}\text { C } & -2.3701698427 & -3.1005676163 & -2.3004606223\end{array}$

$\begin{array}{lllll}\text { O } & -2.2223685162 & -2.3673902477 & -1.2509361104\end{array}$

$\begin{array}{llll}\text { O } & -1.9735193594 & -4.2958737311 & -2.2994987415\end{array}$

$\begin{array}{llll}\text { C } & 2.2398000000 & -1.3683000001 & -4.8790000000\end{array}$

$\begin{array}{llll}\mathrm{N} & 1.1027387203 & -0.8755748483 & -4.2766491737\end{array}$

$\begin{array}{llll}\text { C } & 2.5658499125 & -2.4963647828 & -4.1701136014\end{array}$

$\begin{array}{lllll}\text { C } & 0.7849541363 & -1.7000129919 & -3.2472784040\end{array}$

$\begin{array}{llll}\mathrm{N} & 1.6540459484 & -2.6955093066 & -3.1504059625\end{array}$ 


\begin{tabular}{|c|c|c|c|}
\hline $\mathrm{Zn}$ & -1.6486774584 & -3.9332729558 & 0.0225673723 \\
\hline $\mathrm{O}$ & 1.5510481707 & -3.1447063154 & 0.3039993950 \\
\hline $\mathrm{O}$ & 0.0850109416 & -4.8853300626 & 0.3700203446 \\
\hline $\mathrm{C}$ & 2.3049064510 & -5.3188009621 & 1.1394476609 \\
\hline $\mathrm{H}$ & -0.2790898303 & 0.9984009793 & -0.3397053141 \\
\hline $\mathrm{H}$ & -0.8258504187 & -0.6910778221 & -0.1936079573 \\
\hline $\mathrm{H}$ & -3.0828478431 & -1.1616163180 & 1.0867293740 \\
\hline $\mathrm{H}$ & -0.9281509934 & -3.9820317399 & 3.3443321551 \\
\hline $\mathrm{H}$ & -1.6052811656 & -8.0393610905 & 2.5201909067 \\
\hline $\mathrm{H}$ & -0.3898764704 & -6.0289343436 & 1.9706440325 \\
\hline $\mathrm{H}$ & -4.1606896237 & -2.7342509730 & -3.4048211844 \\
\hline $\mathrm{H}$ & -2.7367374283 & -2.9869498505 & -4.4288442847 \\
\hline $\mathrm{H}$ & 3.3964450595 & -3.1695484828 & -4.3326465137 \\
\hline $\mathrm{H}$ & 1.6678818373 & -4.0220774272 & -1.9057541613 \\
\hline $\mathrm{H}$ & -0.0718224438 & -1.5439949848 & -2.6081040341 \\
\hline $\mathrm{H}$ & -3.8053796118 & -5.6493763658 & -1.6849722843 \\
\hline $\mathrm{H}$ & -3.1679930445 & -5.6587481776 & 2.4680478576 \\
\hline $\mathrm{H}$ & 2.2455415687 & -6.3941970420 & 0.9475554911 \\
\hline $\mathrm{H}$ & 1.9953967747 & -5.1360047971 & 2.1709977317 \\
\hline $\mathrm{H}$ & 3.3461894780 & -5.0002950184 & 1.0179226608 \\
\hline $\mathrm{H}$ & 0.5934360875 & -0.0454129140 & -4.5523992937 \\
\hline $\mathrm{H}$ & -5.2065483711 & -7.1130829022 & 2.0064827391 \\
\hline $\mathrm{H}$ & 0.8664918665 & -0.3510793410 & -0.5701846875 \\
\hline $\mathrm{H}$ & 2.6963064869 & -0.8865872618 & -5.7307559758 \\
\hline $\mathrm{H}$ & 0.1176518167 & -8.1331882412 & 2.9686949110 \\
\hline $\mathrm{H}$ & -1.0964594005 & -7.7026323466 & 4.1901978732 \\
\hline $\mathrm{H}$ & -2.9764865351 & -0.3002609291 & 3.7224657113 \\
\hline
\end{tabular}




$\begin{array}{llll}\mathrm{H} & -1.6256271622 & -2.1100816740 & 4.9181686047 \\ \mathrm{H} & -5.7461558617 & -7.2353199394 & -0.4873585619 \\ \mathrm{C} & 1.3471006551 & -4.5628206433 & 0.2136457433 \\ \mathrm{C} & 1.1489107934 & -6.0476123037 & -1.9306143449 \\ \mathrm{H} & 0.0868565485 & -5.8276989001 & -2.0167264595 \\ \mathrm{H} & 1.5799281037 & -6.2008065704 & -2.9221728008 \\ \mathrm{H} & 1.3026218969 & -6.9381838196 & -1.3197725084 \\ \mathrm{~N} & 1.8193388022 & -4.8789722094 & -1.2996432083 \\ \mathrm{H} & 2.8306438644 & -5.0352856229 & -1.2925554178 \\ \mathrm{H} & 0.8862764533 & -1.8171435962 & 1.3664022107 \\ \mathrm{H} & -2.9693865996 & -1.4352066787 & -3.5555260488 \\ \mathrm{H} & 2.4995739854 & -2.9487784772 & 0.3701797978\end{array}$

\section{TS3}

\begin{tabular}{|c|c|c|c|}
\hline \multicolumn{2}{|c|}{ Solution phase energy: } & \multicolumn{2}{|c|}{-1643.02950424175} \\
\hline $\mathrm{C}$ & 0.2917950000 & 0.0722880001 & 0.2851869999 \\
\hline $\mathrm{r}$ & 0.7141399549 & -0.4321191733 & 1.6480094048 \\
\hline $\mathrm{O}$ & 0.6534095929 & 0.2234002003 & 2.6681495099 \\
\hline & 1.1953181636 & -1.6900657778 & 1.6879640149 \\
\hline & -2.5886630000 & -1.0803560001 & 2.9851679999 \\
\hline & -1.8544201408 & -1.9819490121 & 3.7281842002 \\
\hline & -2.7004098990 & -1.6362244703 & 1.7400827230 \\
\hline 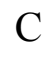 & -1.5346840756 & -3.0277469809 & 2.9380936974 \\
\hline v & -2.0415883100 & -2.8572101783 & 1.7206807991 \\
\hline & -0.5363940000 & -7.3928160000 & 3.1496860000 \\
\hline & -0.6260311010 & -6.0154160069 & 2.8162465686 \\
\hline & -5.0911280000 & -6.8558110000 & 0.1173800000 \\
\hline
\end{tabular}




\begin{tabular}{|c|c|c|c|}
\hline $\mathrm{N}$ & -4.6894537327 & -6.8476775252 & 1.4381349340 \\
\hline $\mathrm{C}$ & -4.2430091677 & -6.0041458155 & -0.5336728385 \\
\hline $\mathrm{C}$ & -3.6368009597 & -6.0109780308 & 1.5553679741 \\
\hline $\mathrm{N}$ & -3.3408431013 & -5.4791541934 & 0.3743825044 \\
\hline $\mathrm{C}$ & -2.9502640000 & -2.6087250000 & -3.6617140000 \\
\hline $\mathrm{C}$ & -2.4137714078 & -3.1753868756 & -2.3618211727 \\
\hline $\mathrm{O}$ & -2.2776508890 & -2.4085937738 & -1.3460025563 \\
\hline $\mathrm{O}$ & -2.1394324302 & -4.4076975523 & -2.2691432634 \\
\hline $\mathrm{C}$ & 2.3092629999 & -1.2485710000 & -4.7930649999 \\
\hline $\mathrm{N}$ & 1.0807422063 & -0.7946663849 & -4.3612550507 \\
\hline $\mathrm{C}$ & 2.6075915643 & -2.3186347857 & -3.9924280347 \\
\hline $\mathrm{C}$ & 0.6815271671 & -1.5808075558 & -3.3364476115 \\
\hline $\mathrm{N}$ & 1.5849278624 & -2.5230943080 & -3.0834626886 \\
\hline $\mathrm{Zn}$ & -1.8284952678 & -4.0423160627 & -0.0437289150 \\
\hline $\mathrm{O}$ & 0.9299141359 & -3.3018120225 & -0.5795786394 \\
\hline $\mathrm{O}$ & -0.1004524803 & -5.1149218353 & 0.2399812815 \\
\hline $\mathrm{C}$ & 2.2909939839 & -5.0722484765 & 0.4213123883 \\
\hline $\mathrm{H}$ & -0.0188255856 & 1.1147705439 & 0.3643694119 \\
\hline $\mathrm{H}$ & -0.5395477143 & -0.5270445389 & -0.1010168820 \\
\hline $\mathrm{H}$ & -3.1904949678 & -1.2501126024 & 0.8591715221 \\
\hline $\mathrm{H}$ & -0.9668375142 & -3.8867777064 & 3.2663023305 \\
\hline $\mathrm{H}$ & -1.2518576036 & -8.0084404938 & 2.5850833389 \\
\hline $\mathrm{H}$ & -0.3541425239 & -5.8909426561 & 1.8849741462 \\
\hline $\mathrm{H}$ & -4.0232669064 & -2.8295953546 & -3.7182589580 \\
\hline $\mathrm{H}$ & -2.4691194899 & -3.0852415137 & -4.5192355786 \\
\hline $\mathrm{H}$ & 3.4870974913 & -2.9466996459 & -4.0137423141 \\
\hline $\mathrm{H}$ & 0.9959480238 & -4.6464336154 & -2.5728787769 \\
\hline
\end{tabular}




\begin{tabular}{|c|c|c|c|}
\hline $\mathrm{H}$ & -0.2454709693 & -1.4397689506 & -2.7987738120 \\
\hline $\mathrm{H}$ & -4.2012891064 & -5.7358094097 & -1.5778472244 \\
\hline $\mathrm{H}$ & -3.0939489999 & -5.8366071546 & 2.4725303681 \\
\hline $\mathrm{H}$ & 2.3614572199 & -6.1594200073 & 0.5178199667 \\
\hline $\mathrm{H}$ & 2.2664565275 & -4.6397824830 & 1.4262340407 \\
\hline $\mathrm{H}$ & 3.1763747897 & -4.6910004795 & -0.0956864650 \\
\hline $\mathrm{H}$ & 0.5693224650 & -0.0060274832 & -4.7386546217 \\
\hline $\mathrm{H}$ & -5.1096729393 & -7.3742467486 & 2.1940525006 \\
\hline $\mathrm{H}$ & 1.1239849405 & -0.0061816371 & -0.4229864025 \\
\hline $\mathrm{H}$ & 2.8410992056 & -0.7820360186 & -5.6087999094 \\
\hline $\mathrm{H}$ & 0.4723107641 & -7.7969528877 & 2.9847979642 \\
\hline $\mathrm{H}$ & -0.7680625473 & -7.4957865997 & 4.2143285415 \\
\hline $\mathrm{H}$ & -2.9406315291 & -0.1462383498 & 3.3956242375 \\
\hline $\mathrm{H}$ & -1.5292352191 & -1.8470049904 & 4.6774639698 \\
\hline $\mathrm{H}$ & -5.9222586396 & -7.4528762141 & -0.2265509929 \\
\hline $\mathrm{C}$ & 1.0006500222 & -4.6755843688 & -0.2856371103 \\
\hline $\mathrm{C}$ & 0.3341162713 & -6.5727215756 & -2.1269151802 \\
\hline $\mathrm{H}$ & -0.7053954914 & -6.2541256163 & -2.1402710001 \\
\hline $\mathrm{H}$ & 0.6004869555 & -7.0210964999 & -3.0874824217 \\
\hline $\mathrm{H}$ & 0.4941827794 & -7.2948518806 & -1.3260364846 \\
\hline $\mathrm{N}$ & 1.1885815345 & -5.3862921885 & -1.8879360620 \\
\hline $\mathrm{H}$ & 2.1724242743 & -5.6346652838 & -2.0085695811 \\
\hline $\mathrm{H}$ & 1.1115615022 & -2.1472448396 & 0.8228237511 \\
\hline $\mathrm{H}$ & -2.8265196013 & -1.5250675618 & -3.7054333115 \\
\hline $\mathrm{H}$ & 1.3918837454 & -3.0290742034 & -1.4239373805 \\
\hline
\end{tabular}




$\begin{array}{lrrr}\text { Solution phase energy: } & -1643.03695045119 \\ \mathrm{C} & 0.0000000000 & 0.0000000000 & 0.0000000000 \\ \mathrm{C} & 0.6097180000 & -0.3281060000 & 1.3471250000 \\ \mathrm{O} & 0.6441310000 & 0.4469410000 & 2.2819740000 \\ \mathrm{O} & 1.1567300000 & -1.5507430000 & 1.4690500000 \\ \mathrm{C} & -2.5764000000 & -1.1808000000 & 3.2435000000 \\ \mathrm{~N} & -1.8194950000 & -2.0906350000 & 3.9529290000 \\ \mathrm{C} & -2.6085450000 & -1.6494260000 & 1.9595130000 \\ \mathrm{C} & -1.4152190000 & -3.0615630000 & 3.1081220000 \\ \mathrm{~N} & -1.8781210000 & -2.8242910000 & 1.8850470000 \\ \mathrm{C} & -0.8189000000 & -7.5844000000 & 3.1388000000 \\ \mathrm{O} & -0.6789480000 & -6.1977260000 & 2.8466380000 \\ \mathrm{C} & -4.9297000000 & -6.6663000000 & -0.0689000000 \\ \mathrm{~N} & -4.6887060000 & -6.6503110000 & 1.2894440000 \\ \mathrm{C} & -3.9900550000 & -5.8516740000 & -0.6279670000 \\ \mathrm{C} & -3.6322790000 & -5.8425850000 & 1.5184260000 \\ \mathrm{~N} & -3.1872980000 & -5.3407020000 & 0.3733570000 \\ \mathrm{C} & -3.0901000000 & -2.5191000000 & -3.5077000000 \\ \mathrm{C} & -2.4011280000 & -3.1412760000 & -2.3045710000 \\ \mathrm{O} & -2.4248400000 & -2.5115980000 & -1.1918920000 \\ \mathrm{O} & -1.8702760000 & -4.2857820000 & -2.4046500000 \\ \mathrm{C} & 2.2398000000 & -1.3683000000 & -4.8790000000 \\ \mathrm{~N} & 0.9987250000 & -0.8073130000 & -4.6752830000 \\ \mathrm{C} & 2.4718290000 & -2.1607280000 & -3.7864390000 \\ \mathrm{C} & 0.5200310000 & -1.2669370000 & -3.4971830000 \\ \mathrm{~N} & 1.3890150000 & -2.0943780000 & -2.9303620000\end{array}$




$\begin{array}{llll}\mathrm{Zn} & -1.6401520000 & -3.9480890000 & 0.1076660000 \\ \mathrm{O} & 0.6816840000 & -3.2038790000 & -0.6517570000 \\ \mathrm{O} & 0.0106680000 & -5.1332290000 & 0.2642950000 \\ \mathrm{C} & 2.3915940000 & -4.8386360000 & -0.0276670000 \\ \mathrm{H} & -0.4497840000 & 0.9927700000 & 0.0402440000 \\ \mathrm{H} & -0.7583830000 & -0.7356410000 & -0.2835390000 \\ \mathrm{H} & -3.0939250000 & -1.2324980000 & 1.0900620000 \\ \mathrm{H} & -0.8258620000 & -3.9191060000 & 3.4010790000 \\ \mathrm{H} & -1.5757810000 & -8.0716970000 & 2.5073670000 \\ \mathrm{H} & -0.2980860000 & -6.0774340000 & 1.9562770000 \\ \mathrm{H} & -4.1042250000 & -2.9313300000 & -3.5773940000 \\ \mathrm{H} & -2.5636360000 & -2.7775640000 & -4.4295620000 \\ \mathrm{H} & 3.3451310000 & -2.7572510000 & -3.5621370000 \\ \mathrm{H} & -0.1686550000 & -4.8691570000 & -2.2616710000 \\ \mathrm{H} & -0.4372040000 & -0.9760960000 & -3.0900910000 \\ \mathrm{H} & -3.8184080000 & -5.6089190000 & -1.6647420000 \\ \mathrm{H} & -3.2031560000 & -5.6620190000 & 2.4918970000 \\ \mathrm{H} & 2.5913240000 & -5.9072720000 & 0.0804740000 \\ \mathrm{H} & 2.5211740000 & -4.3658710000 & 0.9494690000 \\ \mathrm{H} & 3.1177270000 & -4.4013520000 & -0.7202760000 \\ \mathrm{H} & 0.5310050000 & -0.1527170000 & -5.2906000000 \\ \mathrm{H} & -5.2127240000 & -7.1514780000 & 1.9960020000 \\ \mathrm{H} & 0.7824420000 & -0.0101030000 & -0.7670640000 \\ \mathrm{H} & 2.8324440000 & -1.1507100000 & -5.7550290000 \\ & 0.1276340000 & -8.1317540000 & 3.0280280000 \\ \mathrm{H} & -1.13925800000 & -7.6713900000 & 4.18159600000 \\ \mathrm{H} & -3.0043840000 & -0.3037370000 & 3.7047470000\end{array}$




$\begin{array}{llll}\mathrm{H} & -1.5820760000 & -2.0328260000 & 4.9355960000 \\ \mathrm{H} & -5.7311240000 & -7.2467370000 & -0.5008030000 \\ \mathrm{C} & 0.9551470000 & -4.5908600000 & -0.4749670000 \\ \mathrm{C} & 0.9081010000 & -6.6487970000 & -2.1061170000 \\ \mathrm{H} & 0.2990560000 & -7.0949820000 & -1.3204840000 \\ \mathrm{H} & 0.54686440000 & -6.9625980000 & -3.0871840000 \\ \mathrm{H} & 1.9511580000 & -6.9437700000 & -1.9865690000 \\ \mathrm{~N} & 0.7890020000 & -5.1714240000 & -1.9909690000 \\ \mathrm{H} & 1.4380710000 & -4.7109590000 & -2.6338740000 \\ \mathrm{H} & 1.0111080000 & -2.1091560000 & 0.6681570000 \\ \mathrm{H} & -3.1741000000 & -1.4357130000 & -3.4018210000 \\ \mathrm{H} & 1.0542040000 & -2.7915210000 & -1.5143130000\end{array}$

\section{TS4}

\begin{tabular}{|c|c|c|c|}
\hline \multicolumn{2}{|c|}{ Solution phase energy: } & \multicolumn{2}{|c|}{-1643.03124092637} \\
\hline $\mathrm{C}$ & 0.0000000000 & 0.0000000000 & 0.0000000000 \\
\hline $\mathrm{C}$ & 0.5395797405 & -0.3261309193 & 1.3787814090 \\
\hline & 0.4206981780 & 0.3905216588 & 2.3496100875 \\
\hline & 1.1949240713 & -1.5027537969 & 1.4885251204 \\
\hline & -2.5764000000 & -1.1808000000 & 3.2435000000 \\
\hline & -1.7360335730 & -2.0490164398 & 3.9086749102 \\
\hline 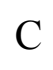 & -2.6789211754 & -1.6679159247 & 1.9704870743 \\
\hline $\mathrm{C}$ & -1.3485864152 & -3.0124596117 & 3.0481610521 \\
\hline & -1.9072598593 & -2.8144123109 & 1.8583176989 \\
\hline & -0.8189000000 & -7.5844000000 & 3.1388000000 \\
\hline & -0.8025462047 & -6.1918201234 & 2.8461867654 \\
\hline & -4.9297000000 & -6.6663000000 & -0.0689000000 \\
\hline
\end{tabular}




\begin{tabular}{llll}
$\mathrm{N}$ & -4.6598873871 & -6.6564813231 & 1.2839261699 \\
$\mathrm{C}$ & -4.0164449906 & -5.8332343570 & -0.6430132292 \\
$\mathrm{C}$ & -3.6122192865 & -5.8338718219 & 1.4965182403 \\
$\mathrm{~N}$ & -3.1994882390 & -5.3179204796 & 0.3449486798 \\
$\mathrm{C}$ & -3.0901000000 & -2.5191000000 & -3.5077000000 \\
$\mathrm{C}$ & -2.4296453998 & -3.0436409955 & -2.2466564220 \\
$\mathrm{O}$ & -2.4239308267 & -2.3268775897 & -1.1895736402 \\
$\mathrm{O}$ & -1.9299517403 & -4.2102714321 & -2.2259524097 \\
$\mathrm{C}$ & 2.2398000000 & -1.3683000000 & -4.8790000000 \\
$\mathrm{~N}$ & 0.9868441474 & -0.8840013859 & -4.5784959697 \\
$\mathrm{C}$ & 2.5722611346 & -2.2082320294 & -3.8510915359 \\
$\mathrm{C}$ & 0.5997369015 & -1.4314666184 & -3.4049731807 \\
$\mathrm{~N}$ & 1.5400209598 & -2.2436838962 & -2.9365823912 \\
$\mathrm{Zn}$ & -1.6908585888 & -3.8897829514 & 0.0513926118 \\
$\mathrm{O}$ & 0.7891659544 & -3.2654697016 & -0.6938548226 \\
$\mathrm{O}$ & 0.0192838392 & -5.0582145669 & 0.3457407590 \\
$\mathrm{C}$ & 2.3992970010 & -5.0067471280 & -0.0775819263 \\
$\mathrm{H}$ & -0.3964854700 & 1.0161783344 & 0.0054823356 \\
$\mathrm{H}$ & -0.7987439337 & -0.6965896594 & -0.2740382708 \\
$\mathrm{H}$ & -3.2300487823 & -1.2777855411 & 1.1283186211 \\
$\mathrm{H}$ & -0.7102087614 & -3.8420069278 & 3.3142522381 \\
$\mathrm{H}$ & -1.4126361539 & -8.1573455837 & 2.4122254112 \\
$\mathrm{H}$ & -0.3568297682 & -6.0479147630 & 1.9930053407 \\
$\mathrm{H}$ & -4.1081996965 & -2.9239387585 & -3.5569362358 \\
$\mathrm{H}$ & -2.5553607819 & -2.8615447523 & -4.3967735398 \\
\hline & -0.1835074066 & -4.9752694710 & -2.3885264450
\end{tabular}




\begin{tabular}{|c|c|c|c|}
\hline $\mathrm{H}$ & -0.3420776319 & -1.2126424284 & -2.9243994877 \\
\hline $\mathrm{H}$ & -3.8701042465 & -5.5776860126 & -1.6800970444 \\
\hline $\mathrm{H}$ & -3.1465068363 & -5.6699289297 & 2.4558323218 \\
\hline $\mathrm{H}$ & 2.4652473002 & -6.0916095239 & 0.0203451474 \\
\hline $\mathrm{H}$ & 2.7051551308 & -4.5568540999 & 0.8741752593 \\
\hline $\mathrm{H}$ & 3.0840632756 & -4.6611224671 & -0.8551800061 \\
\hline $\mathrm{H}$ & 0.4555764223 & -0.2215686488 & -5.1298315329 \\
\hline $\mathrm{H}$ & -5.1568963038 & -7.1759119841 & 1.9964391687 \\
\hline $\mathrm{H}$ & 0.7945523617 & -0.0758221989 & -0.7496007267 \\
\hline $\mathrm{H}$ & 2.7713809749 & -1.0746611950 & -5.7715665208 \\
\hline $\mathrm{H}$ & 0.1917615842 & -8.0141218923 & 3.1754360063 \\
\hline $\mathrm{H}$ & -1.2742175808 & -7.7087203091 & 4.1256163150 \\
\hline $\mathrm{H}$ & -2.9942522751 & -0.3067408561 & 3.7186778872 \\
\hline $\mathrm{H}$ & -1.4015393057 & -1.9435060181 & 4.8583484034 \\
\hline $\mathrm{H}$ & -5.7328653734 & -7.2537966613 & -0.4874311306 \\
\hline $\mathrm{C}$ & 0.9753130570 & -4.5781104229 & -0.3490723343 \\
\hline $\mathrm{C}$ & 0.6399229665 & -6.8546162137 & -2.1015993038 \\
\hline $\mathrm{H}$ & -0.0012885427 & -7.1411122557 & -1.2643873145 \\
\hline $\mathrm{H}$ & 0.2215727330 & -7.2668914177 & -3.0261414856 \\
\hline $\mathrm{H}$ & 1.6352314745 & -7.2781337733 & -1.9468030117 \\
\hline $\mathrm{N}$ & 0.7215834321 & -5.3873447360 & -2.1377638476 \\
\hline $\mathrm{H}$ & 1.4090997127 & -5.0713308751 & -2.8205719762 \\
\hline $\mathrm{H}$ & 1.1372327416 & -2.0207614214 & 0.6600705299 \\
\hline $\mathrm{H}$ & -3.1622388409 & -1.4301382113 & -3.4935239817 \\
\hline $\mathrm{H}$ & 1.2468250457 & -2.9402500599 & -1.5644684809 \\
\hline
\end{tabular}


Solution phase energy: $\quad-1643.05116572151$

$\begin{array}{lllll}\text { C } & 0.8559000001 & -2.28809999997 & -1.5086000008\end{array}$

$\begin{array}{lllll}\text { C } & 0.6897927833 & -3.5995336973 & -0.7701950245\end{array}$

$\begin{array}{lllll}\text { O } & 1.4915062241 & -4.5099968838 & -0.8135633258\end{array}$

$\begin{array}{llll}\text { O } & -0.4480143614 & -3.7356033161 & -0.0667044696\end{array}$

$\begin{array}{llll}\text { C } & 3.0048999972 & -2.68209999986 & 2.2034000004\end{array}$

$\begin{array}{llll}\mathrm{N} & 2.1589644241 & -3.4194425287 & 3.0060334441\end{array}$

$\begin{array}{llll}\text { C } & 2.2612944944 & -1.6197986950 & 1.7658487370\end{array}$

$\begin{array}{llll}\text { C } & 0.9559744969 & -2.8080263549 & 3.0357150728\end{array}$

$\begin{array}{llll}\mathrm{N} & 0.9808132198 & -1.7073609103 & 2.2900305195\end{array}$

$\begin{array}{lllll}\text { C } & -2.34309999997 & -2.05809999993 & 6.09139999957\end{array}$

$\begin{array}{llll}\text { O } & -1.5821489736 & -2.3718233892 & 4.9319327022\end{array}$

$\begin{array}{llll}\text { C } & -0.1441000003 & 2.6498999983 & 5.0763999978\end{array}$

$\begin{array}{llll}\mathrm{N} & 0.1415564288 & 1.4897302621 & 5.7674426222\end{array}$

$\begin{array}{llll}\text { C } & -0.4164795191 & 2.2639586569 & 3.7957465838\end{array}$

$\begin{array}{llll}\text { C } & 0.0419332046 & 0.4527335030 & 4.9087576641\end{array}$

$\begin{array}{llll}\mathrm{N} & -0.2934068071 & 0.8903441105 & 3.7011938776\end{array}$

$\begin{array}{llll}\text { C } & 0.0439000006 & 2.8819000009 & -0.6086000004\end{array}$

$\begin{array}{lllll}\text { C } & -0.2638604734 & 1.6732783727 & 0.2509973578\end{array}$

$\begin{array}{lllll}\text { O } & 0.6060752385 & 0.7448957410 & 0.3649929850\end{array}$

$\begin{array}{llll}\mathrm{O} & -1.3564630512 & 1.6131925556 & 0.8917712500\end{array}$

$\begin{array}{lllll}\text { C } & -3.3957999980 & 0.1611999984 & -4.0476999927\end{array}$

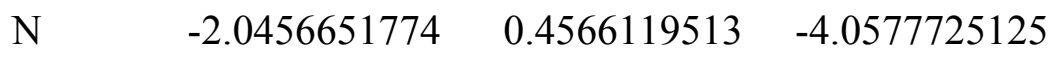

$\begin{array}{llll}\text { C } & -3.6677254826 & -0.3555674631 & -2.8138818046\end{array}$

$\begin{array}{lllll}\text { C } & -1.5083310924 & 0.1271836210 & -2.8777629022\end{array}$

$\begin{array}{llll}\mathrm{N} & -2.4801261040 & -0.3702606606 & -2.1117032307\end{array}$ 


\begin{tabular}{|c|c|c|c|}
\hline $\mathrm{Zn}$ & -0.5397921242 & -0.2296342173 & 1.9180075990 \\
\hline $\mathrm{O}$ & -1.8803995717 & -1.4620218831 & 0.3561495684 \\
\hline $\mathrm{O}$ & -2.3577129781 & -1.2245022003 & 2.4914401104 \\
\hline $\mathrm{C}$ & -3.9542532551 & -2.4294851682 & 1.1391802302 \\
\hline $\mathrm{H}$ & 0.7072762964 & -1.4291779224 & -0.8484670695 \\
\hline $\mathrm{H}$ & 0.1124914003 & -2.2431142527 & -2.3132897049 \\
\hline $\mathrm{H}$ & 2.5464585737 & -0.8043519335 & 1.1188405914 \\
\hline $\mathrm{H}$ & 2.3873347800 & -4.2851587111 & 3.4790974010 \\
\hline $\mathrm{H}$ & 0.1156130996 & -3.1598741748 & 3.6173630879 \\
\hline $\mathrm{H}$ & -2.4111842069 & -0.9743557999 & 6.2644348958 \\
\hline $\mathrm{H}$ & -2.0411399719 & -2.0048321717 & 4.1547519668 \\
\hline $\mathrm{H}$ & 0.4650716301 & 3.6646766817 & 0.0341547662 \\
\hline $\mathrm{H}$ & -0.8674299798 & 3.2808726794 & -1.0604968609 \\
\hline $\mathrm{H}$ & -4.5921699632 & -0.6928173057 & -2.3742866856 \\
\hline $\mathrm{H}$ & -3.4358961315 & 1.3511429128 & 0.2920565450 \\
\hline $\mathrm{H}$ & -0.4722974123 & 0.2477255651 & -2.6018917331 \\
\hline $\mathrm{H}$ & -0.7053595347 & 2.8594878828 & 2.9445641010 \\
\hline $\mathrm{H}$ & 0.1802377643 & -0.5818495849 & 5.1849145262 \\
\hline $\mathrm{H}$ & -2.3526223371 & -0.7348512249 & -1.1429490756 \\
\hline $\mathrm{H}$ & -4.4478817049 & -2.6428226154 & 2.0879019065 \\
\hline $\mathrm{H}$ & -3.7546152763 & -3.3615797749 & 0.6015631219 \\
\hline $\mathrm{H}$ & -4.6115292845 & -1.8111523059 & 0.5181485434 \\
\hline $\mathrm{H}$ & -0.1298506861 & 3.6189084364 & 5.5521494199 \\
\hline $\mathrm{H}$ & 0.3871304305 & 1.4220877116 & 6.7472248818 \\
\hline $\mathrm{H}$ & -3.3635283397 & -2.4635290299 & 6.0415720471 \\
\hline $\mathrm{H}$ & -1.8415721540 & -2.5105889808 & 6.9524438943 \\
\hline $\mathrm{H}$ & 4.0262495559 & -2.9773195870 & 2.0169017935 \\
\hline
\end{tabular}




$\begin{array}{llll}\mathrm{H} & 1.8527470205 & -2.2446799282 & -1.9490982036 \\ \mathrm{H} & -4.0314357600 & 0.3471227641 & -4.8997338024 \\ \mathrm{C} & -2.6713438976 & -1.6718088079 & 1.3501483172 \\ \mathrm{H} & -0.9708720333 & -2.8998424776 & -0.0458526359 \\ \mathrm{~N} & -4.4048394483 & 1.0891724825 & 0.1067637937 \\ \mathrm{H} & -4.7350044341 & 1.7435413817 & -0.6014343242 \\ \mathrm{C} & -5.1905909510 & 1.2809917023 & 1.3352593824 \\ \mathrm{H} & -5.1854528821 & 2.3074171661 & 1.7348299874 \\ \mathrm{H} & -6.2351591443 & 1.0021535908 & 1.1571708131 \\ \mathrm{H} & -4.8029320256 & 0.6200841601 & 2.1163058992 \\ \mathrm{H} & 0.7837629725 & 2.6479684529 & -1.3766897937 \\ \mathrm{H} & -1.5315433717 & 0.8604354919 & -4.8336692765\end{array}$

\section{$\mathrm{CH}_{3} \mathrm{OH}$}
$\begin{array}{llll}\text { C } & -0.0668348615 & 0.7258901314 & 0.0000000000\end{array}$
$\begin{array}{llll}\text { H } & -0.6029020127 & 1.0796730401 & -0.8916545386\end{array}$
H $\quad 0.9283269773 \quad 1.1796276653 \quad 0.00000000000$
$\begin{array}{llll}\mathrm{H} & -0.6029020127 & 1.0796730401 & 0.8916545386\end{array}$
$\begin{array}{llll}\text { O } & 0.1155150916 & -0.6858207276 & 0.0000000000\end{array}$
$\begin{array}{llll}\mathrm{H} & -0.7600400365 & -1.0975505460 & 0.0000000000\end{array}$ 